\title{
EMISSION REDUCTION OF IC ENGINES BY USING WATER-IN- DIESEL EMULSION AND CATALYTIC CONVERTER
}

\author{
Hrishikesh Sane ${ }^{1}$, Neil Purandare ${ }^{2}$, Omkar Barve $^{3}$, Aakash Todakar $^{4}$ \\ ${ }^{I}$ B.E, Mechanical, M.I.T., Maharastra, India \\ ${ }^{2}$ B.E, Mechanical, M.I.T., Maharastra, India \\ ${ }^{3}$ B.E, Mechanical, M.I.T., Maharastra, India \\ ${ }^{4}$ B.E, Mechanical, M.I.T., Maharastra, India
}

\begin{abstract}
IC engines, exhausting gaseous emissions and particulate matter, have long been regarded as one of the major air pollution sources, particularly in metropolitan areas and their extensive use throughout the world has called for better methods to curb these effects. Major IC engine emissions comprise of $\mathrm{NO}_{x}, \mathrm{SO}_{x}, \mathrm{CO}$ and particulate matter and these result due to incomplete combustion of fuel. Many pre-combustion and post-combustion treatments are implemented currently as emission norms are getting stringent by the day. In this paper, causes of emission formation were studied, for both petrol and diesel engines separately and tests were conducted to compare the results of the implemented methods. For diesel engines, NO $\mathrm{O}_{x}$ was found to be the largest pollutant, while for petrol engines, it was $C O$ and HC. Various methods like EGR were studied and maintaining a low temperature was found to be the solution to reduce $N O_{x}$ in diesel engines and oxidation of $C O$ and HC, for petrol engines. As the objectives of petrol and diesel engines differed, different methods were used considering basic individual requirement. Water/diesel (W/D) emulsified formulations are reported to reduce the emissions of NOx, CO and particulate matter (PM) without compensating the engine's performance. In this research a new kind of emulsion was prepared by mixed surfactant method. In case of petrol engine, catalytic converter was successfully employed to reduce emissions to a great extent. Then performance and emission tests were conducted on a 661cc, multi-fuel (diesel and petrol) single cylinder, 4 stroke water cooled engine, with variable compression ratio. Comparison graphs were plotted to study the effects and effectiveness of the two methods was observed and satisfactory results were obtained.
\end{abstract}

Keywords: IC engines, emissions, $N O_{x}$, Water/diesel emulsion and catalytic converter.

\section{INTRODUCTION}

Internal combustion engines generate undesirable emissions during the combustion process. The pollutants that are exhausted from the internal combustion engines affect the atmosphere and contribute largely to problems such as global warming, smog, acid rain, respiratory hazards etc. These emissions are mostly due to non-stoichiometric combustion, dissociation of nitrogen and impurities in the fuel and air. Exhaust gas emissions of IC engines have been considered a very serious issue regarding air quality and the environment and stricter norms such as Euro 4 or Bharat Stage IV are getting implemented. The main pollutants produced from the exhaust of IC engines are hydrocarbon $\mathrm{HC}$, nitrogen oxides (NOx), particulate matter, black smoke, carbon monoxide, and carbon dioxide. Two major ways of reducing the pollutants are - treatment inside the combustion cylinder and after treatment or treatment outside the combustion cylinder, with the latter being relatively easy to implement. Several researchers have shown that introducing water into diesel fuel to produce emulsified diesel fuel can significantly lower the emissions and thus pollution level of particulate matter and NOx. Similarly catalytic convertor has been found useful in controlling emissions from petrol engine.

\section{EMISSION FORMATION}

Though there is large variation in the percentage distribution of various emissions for petrol and diesel engines, the basic formation principle of emissions like $\mathrm{HC}, \mathrm{NO}_{\mathrm{x}}$ and $\mathrm{CO}$ remain same for both. Unburned $\mathrm{HC}$ emissions originate when fuel escapes combustion due to several processes such as flame quenching in narrow passages present in the combustion chamber and on cold chamber walls, and incomplete oxidation of fuel that is trapped or absorbed in oil film or deposits. NOx is formed by oxidation of molecular nitrogen. During combustion at high flame temperatures, nitrogen and oxygen molecules in the inducted air breakdown into atomic species which react to form NO. Some $\mathrm{NO}_{2}$ is also formed and $\mathrm{NO}$ and $\mathrm{NO}_{2}$ together are called as $\mathrm{NO}_{\mathrm{x}}$. $\mathrm{CO}$ results from incomplete oxidation of fuel carbon, when insufficient oxygen is available to completely oxidize the fuel. $\mathrm{CO}$ rises steeply as the air-fuel $(\mathrm{A} / \mathrm{F})$ ratio is decreased below the stoichiometric $\mathrm{A} / \mathrm{F}$ ratio. [1].

\section{OBJECTIVE}

The aim of this research is to create a new fuel that can be used in a diesel engine as a fuel. The fuel is created by making an emulsion of diesel and water by using a suitable surfactant with stability and homogeneity being the key 
concerns. This emulsion is then used in a laboratory installed diesel engine coupled with an eddy current dynamometer. The emission and performance characteristics of the engine are thus noted down and put in tabular form. Then the engine is run with regular diesel fuel and same observations are taken. A comparison is done between the two cases in terms of performance and emission properties of the fuel.

For petrol engine, select, procure and fabricate a compatible 3-Way Catalytic Converter assembly with the piping and install on the laboratory engine. The emission characteristics of petrol engine are to be studied with and without catalytic converter.

\section{WATER-IN-DIESEL EMULSION}

An emulsion can be defined as a mixture of two liquids in which one is present in droplets of macroscopic or ultramicroscopic size, distributed throughout the other. Emulsions are made from the constituents spontaneously or by a mechanical way. In spontaneous emulsions, the mixing is easy and spontaneous. (Britannica) But if they don't mix properly then a third chemical called a surfactant is used to bind the molecules of the constituent liquids. Surfactants are compounds that lower the surface tension of a liquid that is it decreases the interfacial tension between two liquids, or that between a liquid and a solid.

Water in diesel emulsion comes under the category of water in oil emulsion. The surfactant that has to be used should have an HLB value in the range of 7 to 11 . One surfactant with this value can be used otherwise mixed surfactant can be used to obtain the required HLB value. In the latter case the two surfactants should be chosen carefully so that one is hydrophilic and other is lipophilic [2]. In this experiment, a mixed surfactant is made and has been used for further trials.

\subsection{Preparation}

Components required for making emulsion are: Mechanical agitator, diesel, distilled water, burette, and pipette.

The pipette, burette and container were thoroughly washed and cleaned dry. Diesel was measured in the burette in required volume and poured into container. Now calculated volume of each surfactant (Span-20 and Tween-20) were measured in the pipette and poured into the container. Same is done for water. Now the container is placed under the mechanical agitator and the mixture is thoroughly mixed for about 10- 15 minutes at constant speed. The emulsion thus obtained is checked for stability. Various trials were carried out before obtaining a stable emulsion.

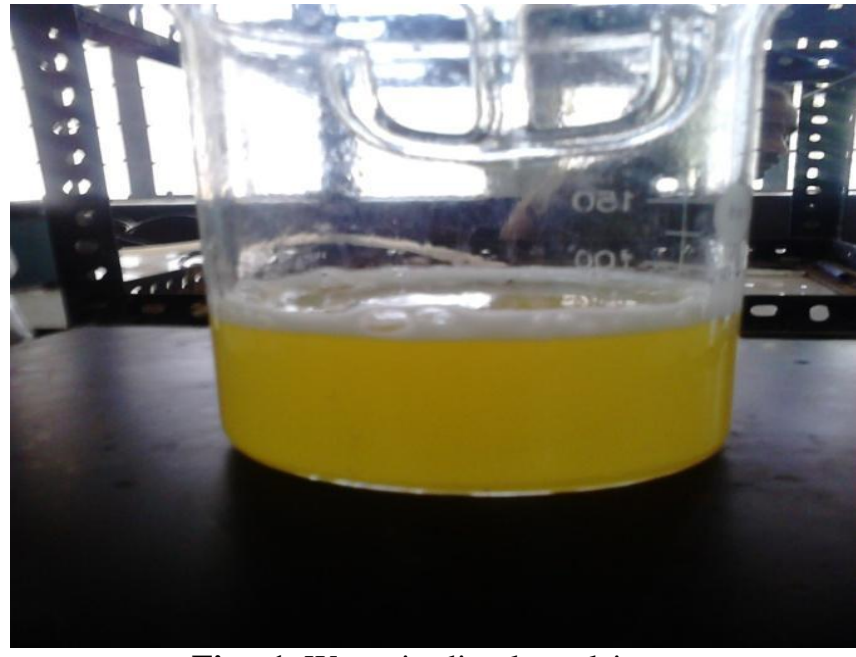

Fig - 1: Water-in-diesel emulsion.

\subsection{Effects on Engine Performance}

It was observed that the brake specific fuel consumption (bsfc) of the engine decreases slightly when an emulsion is used instead of diesel, but it also depends on the concentration of water in the emulsion. It decreases up to a certain limit and then again increases. The bsfc is best obtained for the emulsion with $7.5 \%$ of water.

Also, brake thermal efficiency for emulsions is always higher than that of diesel except at very high loads. Hence, emulsions prove to the better fuel with respect to brake thermal efficiency [3] [4].

\subsection{Effects on Emissions}

As engine performance results were best obtained for emulsion with $7.5 \%$ water, this emulsion was used for further emission tests, and compared with diesel.

a. With increase in load, $\mathrm{CO}$ emission increases for both the fuels used. However, it has been observed that emission of $\mathrm{CO}$ is higher for water-in-diesel emulsion than diesel at most of the loads. This happens because the presence of water decreases the temperature inside the cylinder, slowing down the combustion of carbon, as a result of which incomplete combustion occurs.

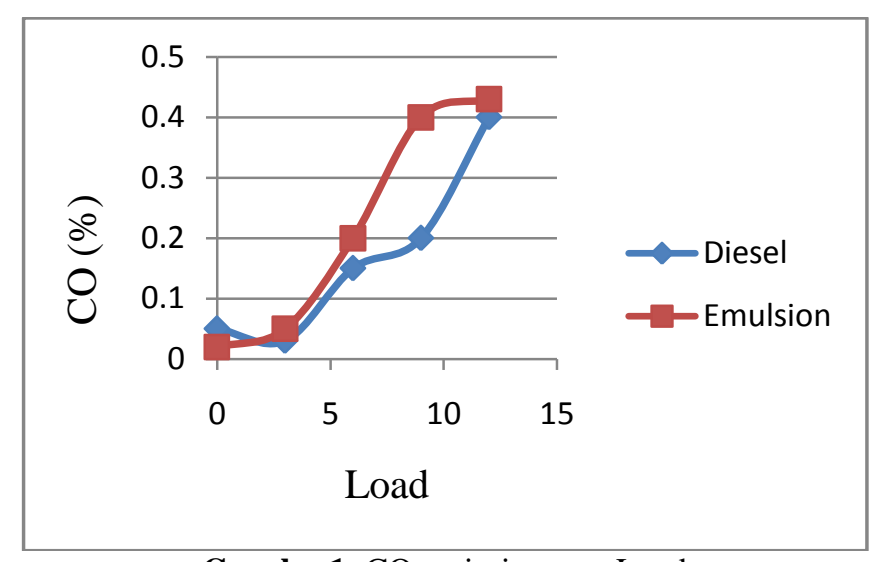

Graph - 1: CO emissions vs. Load 
b. It is seen that $\mathrm{HC}$ emissions increase up to a certain load and then decrease, in case of diesel. For the emulsion, it shows an opposite trend. At low load conditions, HC emissions in case of diesel fuel are more than those of emulsion, but at higher load conditions the emulsion gives more $\mathrm{HC}$ emissions than diesel.

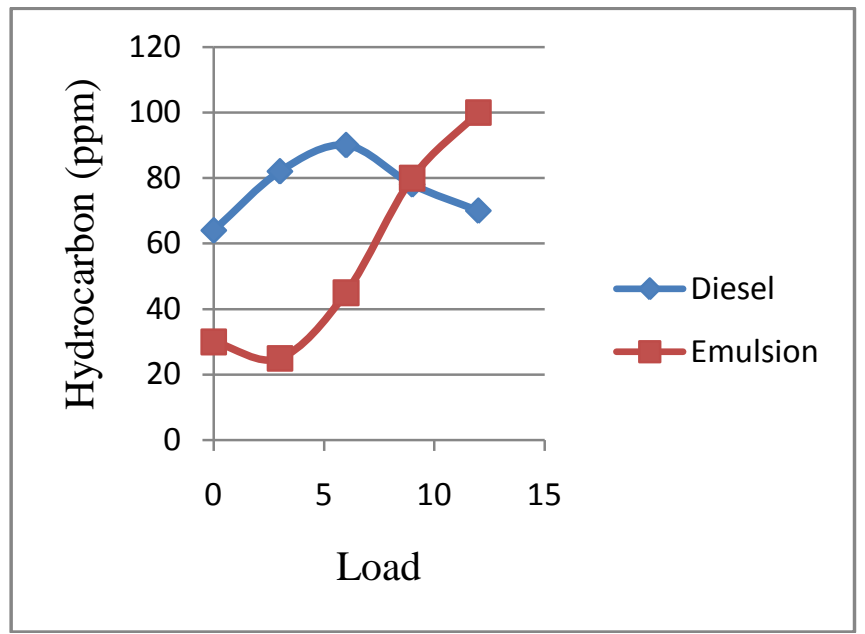

Graph - 2: HC emissions vs. Load.

c. For diesel, $\mathrm{CO}_{2}$ emissions increase almost linearly with increase in load. For the emulsion too it increases linearly with some variations at some loads. $\mathrm{CO}_{2}$ emission increases when we add water to diesel for the same reason of incomplete combustion as happens in $\mathrm{CO}$ emissions.

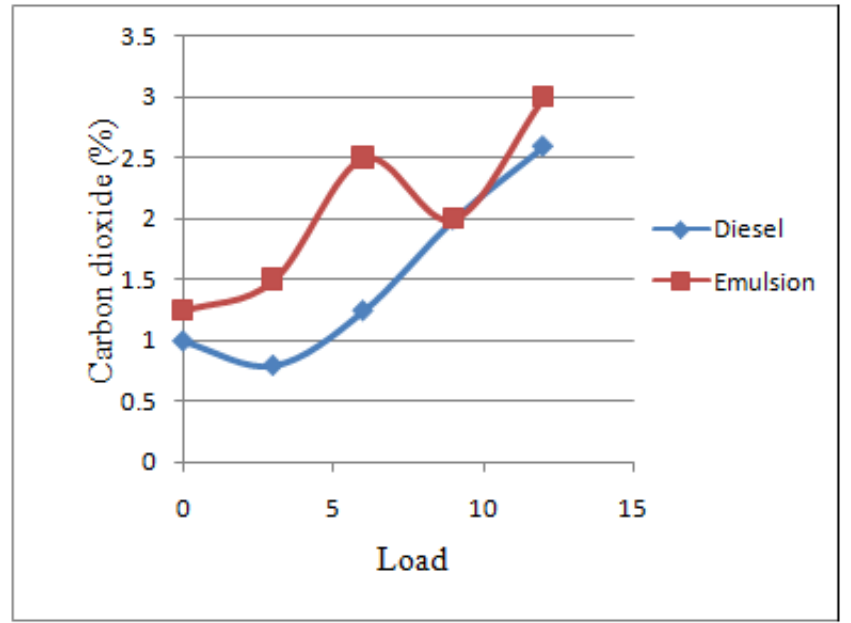

Graph - 3: $\mathrm{CO}_{2}$ emissions vs. Load.

d. With increase in load, NOx emissions increase for both diesel as well as emulsion. It has been observed that using diesel-water emulsion as fuel greatly reduces the NOx emissions as compared to diesel. This happens because when water along with diesel enters the combustion cylinder, it is directly vaporized into steam due to presence of high temperature and pressure inside the cylinder. This takes some of the heat from the combustion chamber and brings down the cylinder temperature. As a result the conversion of diatomic nitrogen to more reactive monoatomic nitrogen decreases thereby reducing the chances of formation of NOx.

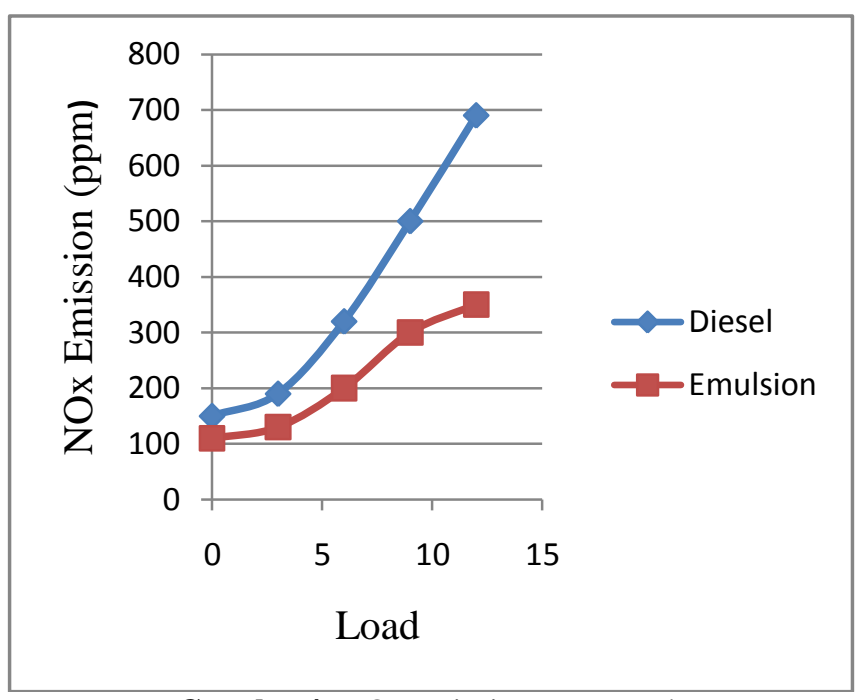

Graph - 4: $\mathrm{NO}_{\mathrm{x}}$ emissions vs. Load.

\section{CATALYTIC CONVERTER}

A three-way catalytic converter has three simultaneous tasks:

1. Reduction of nitrogen oxides to nitrogen and oxygen:

$$
2 \mathrm{NOx} \rightarrow \mathrm{xO}_{2}+\mathrm{N}_{2}
$$

2. Oxidation of carbon monoxide to carbon dioxide:

$$
2 \mathrm{CO}+\mathrm{O}_{2} \rightarrow 2 \mathrm{CO}_{2}
$$

3. Oxidation of unburnt hydrocarbons (HC) to carbon dioxide and water:

$$
\mathrm{CxH}_{2} \mathrm{x}+2+2 \mathrm{xO}_{2} \rightarrow \mathrm{xCO}_{2}+2 \mathrm{xH}_{2} \mathrm{O}
$$

These three reactions occur most efficiently when the catalytic converter receives exhaust from an engine running slightly above the stoichiometric point. This is between 14.6 and 14.8 parts air to 1 part fuel, by weight, for gasoline. The ratio for LPG, natural gas and ethanol fuels is slightly different, requiring modified fuel system settings when using those fuels. Generally, engines fitted with 3-way catalytic converters are equipped with a computerized closed-loop feedback fuel injection system employing one or more oxygen sensors, though early in the deployment of 3-way converters, carburetors equipped for feedback mixture control were used. While a 3-way catalyst can be used in an open-loop system, NOx reduction efficiency is low. Within a narrow fuel/air ratio band surrounding stoichiometry, conversion of all three pollutants is nearly complete. However, outside of that band, conversion efficiency falls off very rapidly. When there is more oxygen than required, then the system is said to be running lean, and the system is in oxidizing condition. In that case, the converter's two oxidizing reactions (oxidation of $\mathrm{CO}$ and hydrocarbons) are favored, at the expense of the reducing reaction. When there is excessive fuel, then the engine is running rich. The reduction of NOx is favored, at the expense of $\mathrm{CO}$ and $\mathrm{HC}$ oxidation [6] [7]. 


\subsection{Installation Procedure}

3 Way Catalytic Converter of a Maruti Suzuki Alto (Capacity-800cc) was purchased from the vendor suited to our engine capacity of approximately 690cc. It was examined for end to end dimensions and possibility of connections in series with the engine exhaust. The engine end of converter was welded with a stainless steel pipe with external threading provided on its free end. This threaded end was fitted to an exhaust flange at engine outlet (Welding technique: Arc welding). The other end of converter was welded with a right angle stainless steel bend which had an internal threading to be fitted to the silencer flange. The rest of the engine assembly was undisturbed. Engine exhaust was directed through the pipe into the converter and from converter exit through the silencer to atmosphere.

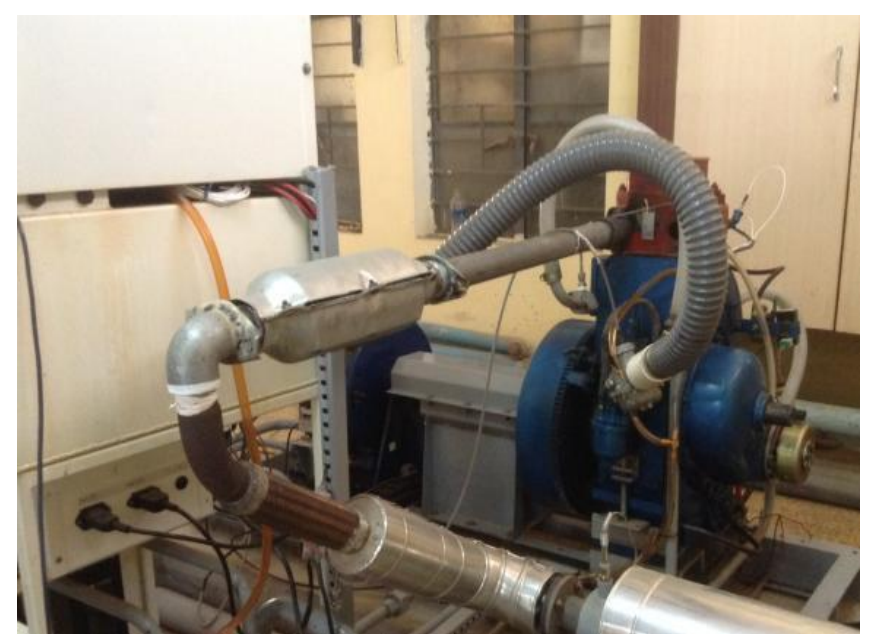

Fig -2: Engine Test Setup - With Catalytic Converter.

\subsection{Experimental Procedure}

The filters of the engine were replaced and the injectors were cleaned and calibrated according to the desired pressure. The AVL gas analyzer was installed. The input to the gas analyzer was taken from the exhaust of the engine. The fuel tank was then filled with petrol and the engine was run. The engine was run at various loads of the dynamometer $-0,3,6,9,12$ and respective readings of gas analyzer were taken for constant compression ratio of 10 without catalytic converter. After all the readings were taken, compression ratio was changed to 9 by indexing procedure provided on the engine and emission readings were taken. Similarly readings were noted for compression ratio 8 . Same steps were taken and the readings were noted down after installing the converter. After taking all the observations graphs were plotted to compare the emission characteristics of the engine with and without catalytic converter and also variation with compression ratio was plotted.

\subsection{Effects on Emissions}

a. The reduction catalyst (Platinum-rhodium) is located in the initial part of the catalytic converter. The temperatures are very important in the reduction reactions for NOx gases and these are obtained closest to the engine exhaust. Also, if the NOx come in contact with the oxidation catalyst first, then there will be further oxidation and increase in their percentages. The reduction catalyst reduces the NOx mainly into gases like $\mathrm{N} 2$ and $\mathrm{O} 2$. The catalyst accelerated reduction reactions require a specific minimum temperature. Due to this, the conversion efficiency is very low at lower temperatures and it is seen to increase as the temperatures are raised. Further, as the gases pass through the oxidation catalyst part, re-oxidation of the gases is reduced due to the absence of required reaction temperatures.

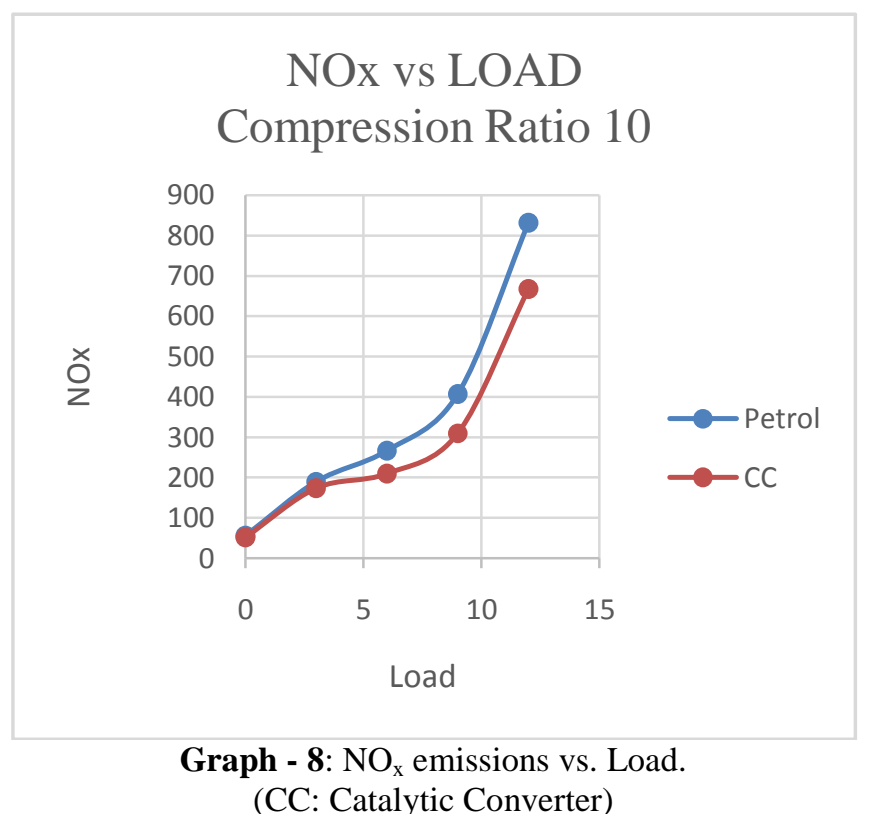

b. The oxidation catalyst (Platinum-Palladium), located in the final part of the converter, accelerates the oxidation of the exhaust $\mathrm{CO}$ into $\mathrm{CO}_{2}$, thereby reducing the $\mathrm{CO}$ emissions. The reduction in $\mathrm{CO}$ is observed to increase with an increase in load on engine. This happens because the temperatures of the exhaust gases increase with load, which helps in further oxidation of the gases.

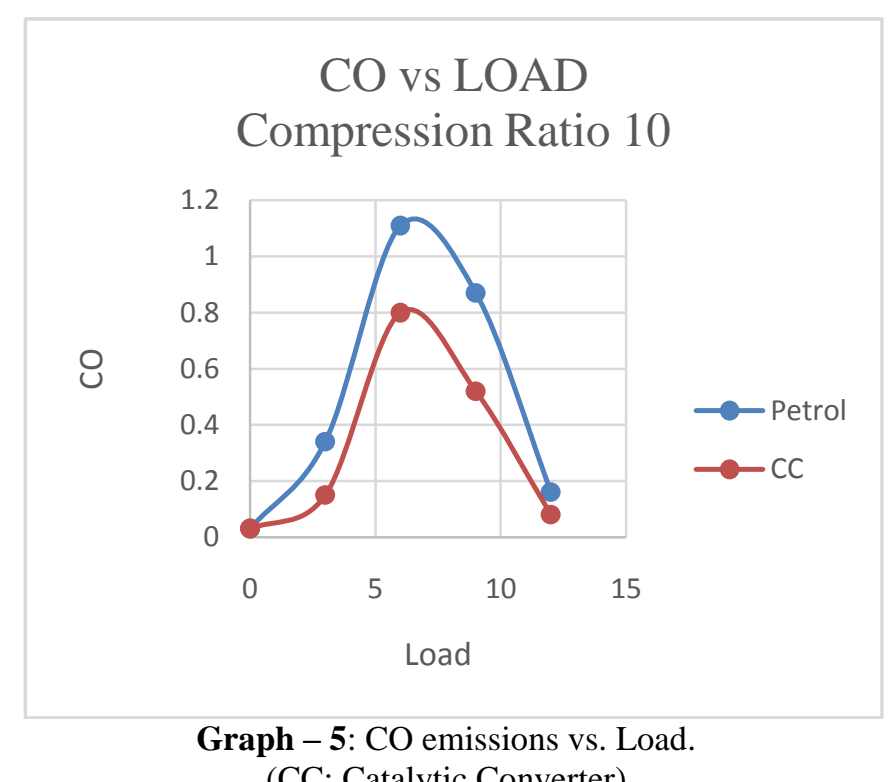


c. The oxidation catalyst (Platinum-Palladium) led to large reduction in engine-out $\mathrm{HC}$ emissions over a wide range of engine operating conditions. The percentage $\mathrm{HC}$ reduction obtained by using catalytic converter is initially low, but increases as the load increases. The increase in load increases the temperature of the exhaust gases which helps the oxidation of $\mathrm{HC}$, hence increasing the conversion efficiency. [8]

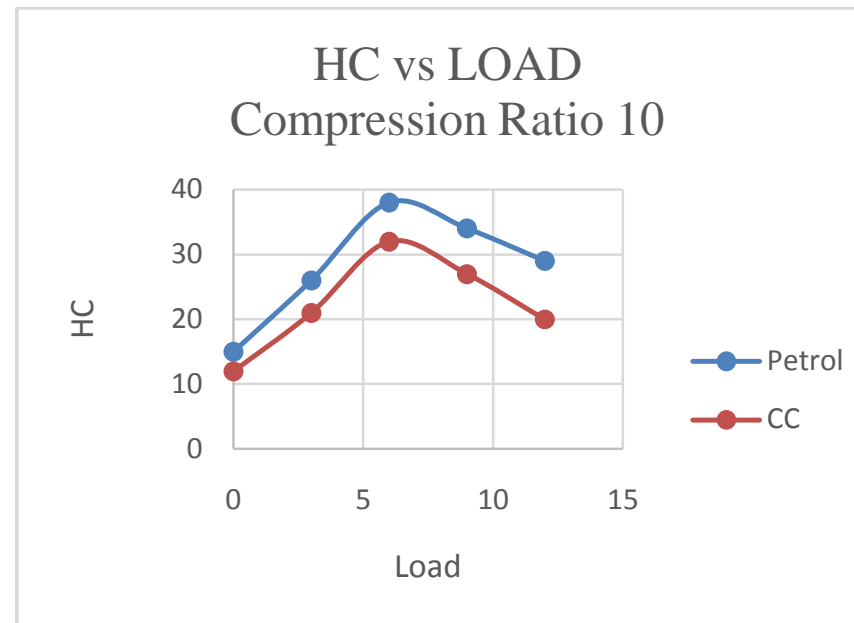

Graph - 6: HC emissions vs. Load.

(CC: Catalytic Converter)

d. $\mathrm{CO}_{2}$ emissions arise due to complete combustion of the fuel and oxidation of $\mathrm{HC}$ and $\mathrm{CO}$ gases. The catalytic converter essentially reduces the $\mathrm{HC}$ and $\mathrm{CO}$ emissions, which results into a higher $\mathrm{CO}_{2}$ emission. It is an indicator of the conversion efficiency of the catalytic converter, and an increase in $\mathrm{CO}_{2}$ indicates a better conversion.

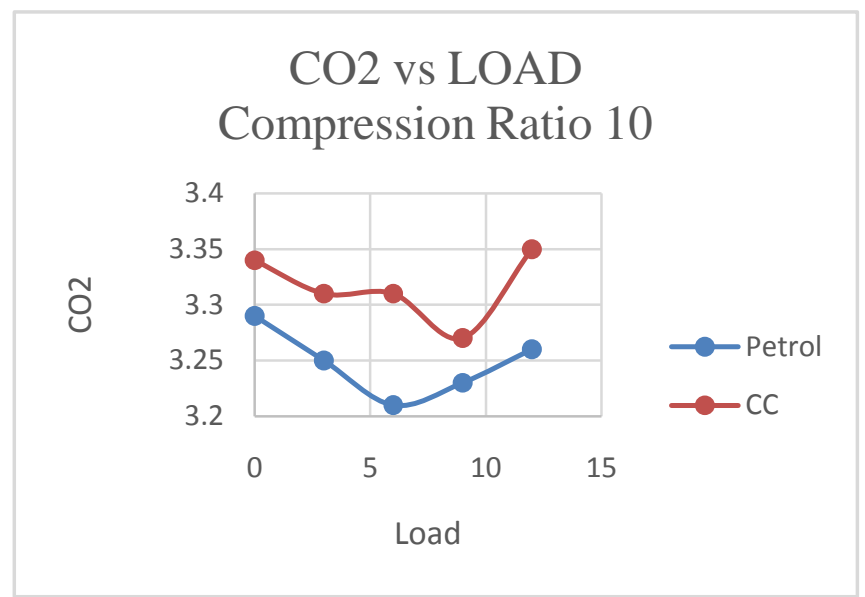

Graph - 7: $\mathrm{CO}_{2}$ emissions vs. Load. (CC: Catalytic Converter)

\section{EFFECT OF COMPRESSION RATIO ON EMISSIONS}

Our test results confirmed the findings on various emissions in relation with compression ratio. If we observe the variation of $\mathrm{CO}$ emissions with Compression Ratio, at majority loads when compression ratio increases $\mathrm{CO}$ emissions increase because as compression ratio increases, thermal efficiency increases which reduces post-flame oxidation reactions of $\mathrm{CO}$, giving rise in $\mathrm{CO}$ emissions. As we increase the compression ratio, the surface to volume ratio increases giving rise to $\mathrm{HC}$ emissions. In addition, crevices volume constitutes a higher fraction of the clearance space and the peak pressures are higher pushing a higher fraction of unburned mixture into the crevices. This leads to increase in $\mathrm{HC}$ emissions as we increase compression ratio. When compression ratio increases, it increases $\mathrm{CO}, \mathrm{HC}$ emissions and temperature also increases giving rise to oxidation of these two, so these two reasons give a mixed trend for $\mathrm{CO}_{2}$ as we increase compression ratio. Also, increase in compression ratio increases the NOx emissions as temperature of burned gases increase when we increase the compression ratio [1].

\section{CONCLUSIONS}

\subsection{Diesel Engine}

The specific fuel consumption was observed to decrease with increase in the percentage of water in diesel. Literature shows that specific fuel consumption is decreased by $2 \%$ to $3 \%$ when concentration of water is increased, but further increase may increase the specific fuel consumption. However at higher loads the fuel consumption is more for emulsions than diesel. The brake thermal efficiency increases with increase in water content emulsion, under low load condition. But it decreases at higher loads. The NOx emission is brought down by $30 \%-50 \%$ by use of diesel water emulsion. This trend goes on increasing with increase in amount of water in the emulsion. At lower loads the hydrocarbon emissions are lesser for emulsion as compared to diesel; however when the load increases HC emissions are higher for emulsions.

Carbon monoxide and carbon dioxide emissions increase with increase in water percentage in the fuel. This is due to the fact that most of the hydrocarbons are burnt at lower loads. According to literature for optimal results use of diesel water emulsion with increasing water content will have the better results in terms of performance and emissions.

\subsection{Petrol Engine}

Catalytic converter has no significant effect on engine operating and performance parameters like b.s.f.c, brake thermal efficiency. Three-way catalytic converters reduce three important emission, carbon monoxide, carbon dioxide and nitrogen oxides, simultaneously. The NOx emission is reduced by $10 \%-20 \%$ by use of 3-way catalytic converter. This trend goes on increasing with increase in temperature of the input engine exhaust gases. The hydrocarbon and carbon monoxide emissions reduce with increasing loads. These are as an effect of improved oxidation reaction conditions due to temperature rise. Carbon dioxide emissions increase with increase in load. This indicates the conversion efficiency of $\mathrm{HC}$ and $\mathrm{CO}$ gases. A higher percentage of carbon dioxide indicates an efficient catalytic converter. Though compression ratio decreases the amount 
of $\mathrm{CO}, \mathrm{HC}$ emissions, but simultaneously decreases thermal efficiency of the engine which is unfavorable. Thus, using 3way catalytic converter is a better option than operating engine at a lower compression ratio.

\section{REFERENCES}

[1]. IC engine combustion and emission by B.P.Pundir.

[2]. Griffin WC: "Classification of Surface-Active Agents by 'HLB,'" Journal of the Society of Cosmetic Chemists 1 (1949): 311.

[3]. C. ALAN CANFIELD “ Effects of diesel water emulsions in diesel engine" thesis submitted in University of Florida, 1999.

[4]. Omar Badrana*, Sadeq Emeishb, Mahmoud Abu-Zaidc, Tayseer Abu-Rahmaa, Mohammad Al-Hasana, Mumin AlRagheba " Impact of Emulsified Water/Diesel Mixture on Engine Performance and Environment" - International journal of thermal and environment engg. (vol 3), 2011.

[5]. Holmberg K, Jönsson B, Kronberg B, Lindman B. Surfactants and Polymers in Aqueous Solution. 2nd ed. Chichester: Wiley; 2003.

[6]. Julie M Pardiwala, Femina Patel, Sanjay Patel "Review paper on Catalytic Converter for Automotive Exhaust Emission" institute of technology, Nirma University, ahmedabad

[7]. http://en.wikipedia.org/wiki/Catalytic_converter.

[8]. V Ganesan, Internal Combustion Engines (third edition)

\section{BIOGRAPHIES}

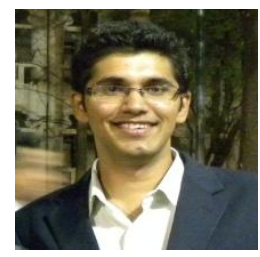

Hrishikesh Sane, born in Maharashtra, India in 1993. He secured his degree in B.E. Mechanical from Maharashtra Institute of Technology, pune, in 2014. Future studies and interest in Design field.

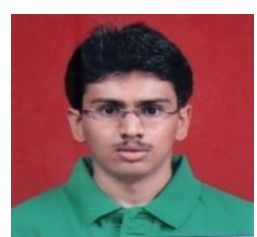

Neil Purandare, born in Maharashtra, India in 1993. He secured his degree in B.E.- Mechanical from Maharashtra Institute of Technology, pune , in 2014. He has completed his project and worked as Intern at varroc polymers pvt. Ltd. He has interest in Future studies and his research interested fields are Design field, Product Design and Development and PLM.

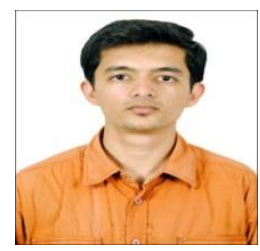

Omkar Barve, born in Maharashtra, India in 1993. He secured his degree in B.E. Mechanical from Maharashtra Institute of Technology, pune , in 2014. Future studies and interest in aerospace field.

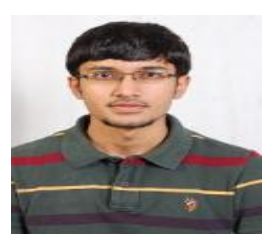

Aakash Todkar, born in Maharashtra, India in 1992. He secured his degree in B.E- Mechanical from Maharashtra Institute of Technology, pune, in 2014. $\mathrm{He}$ is currently working in Bajaj auto. He has interest in Future studies and his interested field is Design field. 